
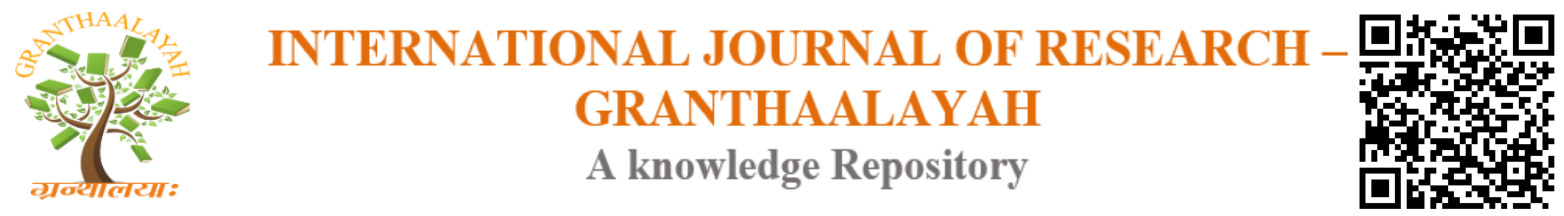

Science

\title{
INTERNET OF THINGS (IOT) BASED ADVANCED ONLINE EXAMINATION USING RASPBERRY PI
}

\author{
Mr.Kunal.S.Pawar ${ }^{* 1}$, Prof.Pravin.C.Latane ${ }^{2}$ \\ ${ }^{* 1,2}$ E\&TC Department, Sinhgad Institute of Technology, Lonavala, India
}

\begin{abstract}
With the development in the education system, considering the latest current online exam system, a new projection of online exam system based on Raspberry pi IOT is proposed, and the key implementation techniques and methods are also described. The growing ubiquity of wireless, RFID mobile and sensor devices has provide a promising opportunity to build the powerful examination systems and applications by Internet of Things (IoT). A wide range of IoT applications have been developed in recent years. In an effort to understand the development of IoT in online examination, here we propose the current research of IoT, IOT key enabling technologies, major IoT applications in online examination and identifies research trends and challenges. Here we initially all the examine details are stored in the server. Then By applying face recognition (in Open CV based) technique, you can start the online examination. Due to sometime unwanted person also enter to wright the exam, so this is the best way to identified any culprits are found or not.
\end{abstract}

Keywords: Online Examination System; Raspberry Pi; IoT; Web Server; Open CV; Python.

Cite This Article: Mr.Kunal.S.Pawar, and Prof.Pravin.C.Latane. (2018). "INTERNET OF THINGS (IOT) BASED ADVANCED ONLINE EXAMINATION USING RASPBERRY PI." International Journal of Research - Granthaalayah, 6(5), 30-36. https:// doi.org/10.29121/granthaalayah.v6.i5.2018.1417.

\section{Introduction}

Then student enter to wright the exam, here also some security is applied. Now a days a papers can be stored online. But here not like that, if you entered to wright the exam, that time only teachers are start to wright questions on server. The examine browser window will print the question paper automatically. So easy avoid the question paper leakage before the exam. As computer technology is constantly evolving, education systems cannot remain firmly devoted to the old traditional ways of learning. The traditional examination procedure requires a great number of human resources, stationery, and investment of time in composing examination questions. In contrast, the new models of online examinations have numerous benefits for both the educator and the students. With online examination, the professors supervise more easily the students' progress and set examinations fluently. Some courses have four examinations per semester, therefore 
without new technology developments, the workload of the examiners would be skyrocketed. Besides, students can take the exam from a distance when they are prepared.

The main purpose of this project was the development of an integrated system that generates examination papers with random questions about the curriculum of digital circuits' courses. This system consists of a database, a website and a questions' program generator. Each examination becomes unique, thanks to the numerous random questions provided by a database of questions, which is produced by a tool program. Furthermore, it should be mentioned that an extension of the website is the anonymous "free exams" concept of the examination tests for students to practice regularly and check the obtained knowledge. Online learning mechanism is a newer and improved version of distance learning. It is a method of delivering education information through the internet. It is not like the traditional education system where time and location matters lot. Through this, a student has the flexibility of not being physically in an academic institute always.

The student can set his/her own pace and choose the most convenient time and place to study. Students around all over the world can learn from these online courses since they do not need to physically go to those educational institutes. When it comes to Higher Education, most of the institutes use Learning Management Systems (LMS) to deliver their course materials to the students. When the students are engaged in online learning, the chance of getting the feedback from a teacher will not be effective, since the teacher does not know 0the emotional state/behavior of the student. Not only for online education systems, when it comes to Game Based Learning or Gamification, there is no way to get the teachers' feedback depending on the student's emotional condition/behavior.

According to Goleman, an expert teacher is able to recognize the emotional state of a student and respond to student in an appropriate way so that it will positively impact on the student's learning process. When it comes to online education, there is no one to monitor the student, and it is basically based on student centered learning mechanism. So the student is unable to get the feedback according to his/her emotional state/behavior. Instead of that the student will get a general feedback from the course coordinator or from the instructor. But that should not be the correct way. Hence there should be a way to identify the emotional state/behavior and give the feedback to the student. The other problem is the tracking of the emotional states/behavior of the students. Since this is for online education systems, the emotional states cannot be detected by using physical devices.

This literature review is carried out to find out how to "learn to learn" and to identify the existing ways and means of evaluating the performance of the students when they are engaged in traditional learning and online learning. Finally there will be a comparison of each and every mechanism.

A examination is "used to practice for the real one" and thus it is taken before an official examination. In such a mock exam the marks do not count, i.e. this "trial examination "does not count for credit. A mock examination is often given to the same standards and under the same controlled conditions as the official examination. Hence, it gives students the opportunity to practice for the later, important examination, to get an idea of the types of questions asked, and to get used to regulations and locations of the official examination.

Furthermore, students can practice to work under time pressure with only those aids allowed to be used in the official examination. Another, even more important aim of mock examinations is to 
provide the students with information on their actual learning progress. Students get an idea of what they still need to learn. Due to the time gap of usually a few days to a few weeks between mock and official examination, there is still the opportunity to fill these knowledge gaps. It is up to the student to use this opportunity.

An electronic mock examination, i.e. online mock exam is a variant that differs significantly from those taken on paper. Electronic mock examinations can be performed unsupervised. Students can take them at home at a time of their choice. In addition, it is way easier to allow students to take electronic mock exams more than once than for the paper-based version. Students take advantage of this opportunity, for instance, to practice only certain parts, e.g. theory questions, of the exam. In all cases of electronic mock examinations, feedback is usually also given electronically. In this work, we describe the setup for an automatically marked electronic mock examination for the course "Basics of Computer Science". We discuss question types other than multiple-choice questions that allow for automated marking.

\section{Materials and Methods}

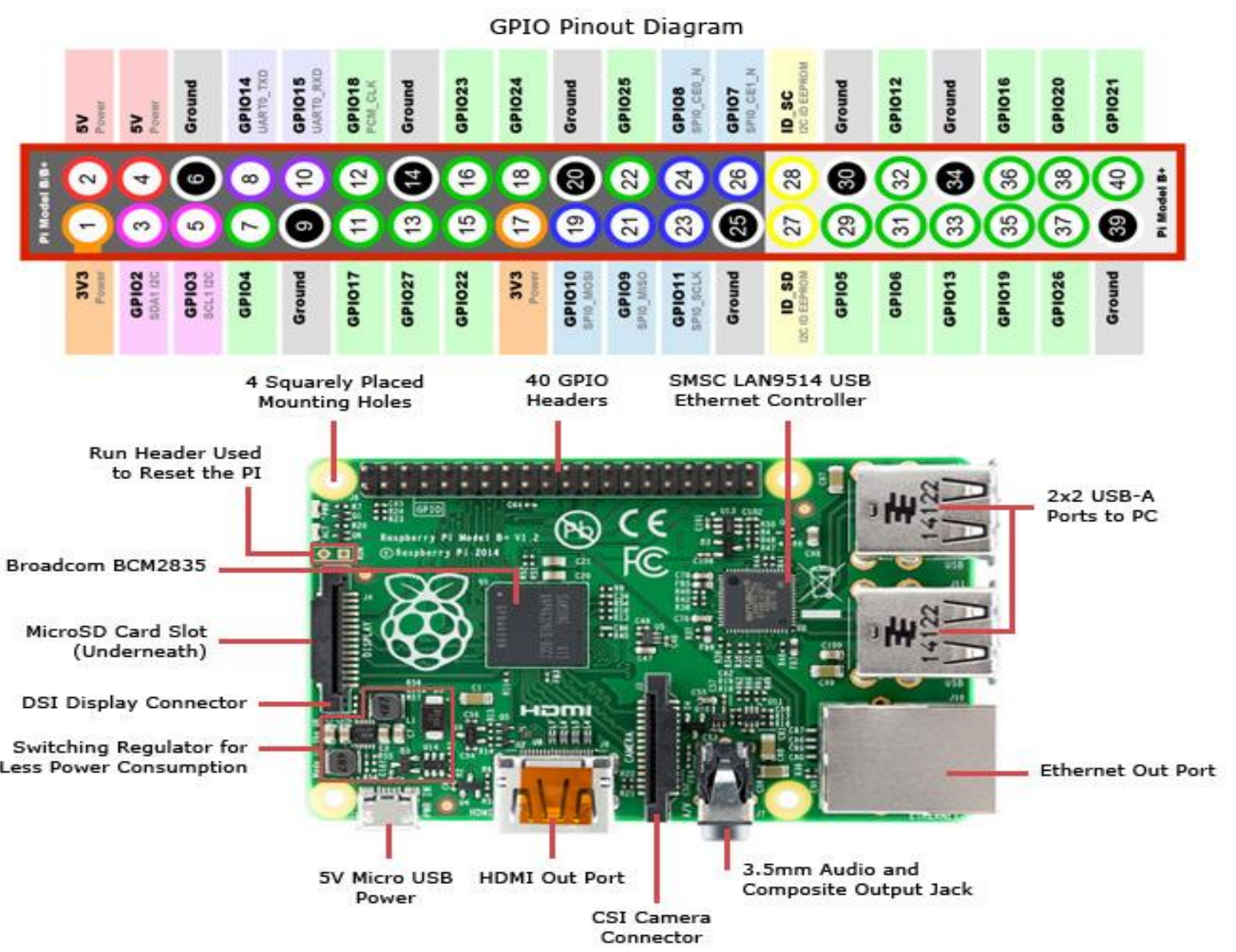

RASPBERRY PI:

Figure 1: Architecture of Raspberry pi

The Raspberry Pi is a credit card sized single-board computer with an open-source platform that has a thriving community of its own, similar to that of the audio. It can be used in various types of projects from beginners learning how to code to hobbyists designing home automation systems. 
There are a few versions of the Raspberry $\mathrm{Pi}$, but the latest version, has improved upon its predecessor in terms of both form and functionality. The Raspberry Pi Model B features:

- $\quad$ More GPIO

- More USB

- Micro SD

- Lower power consumption

- Better audio

- Neater form

Higher-spec variant increases the Raspberry pi GPIO pin count from 26 to 40 pins. There are now four USB 2.0 ports compared to two on the Model B. The SD card slot has been replaced with a more modern push-push type micro SD slot. It consumes slightly less power, provides better audio quality and has a cleaner form factor.

To get started you need a Raspberry Pi 3 Model B, a 5V USB power supply of at least 2 amps with a micro USB cable, any standard USB keyboard and mouse, an HDMI cable and monitor/TV for display, and a micro SD card with the operating system pre-installed.

This pinots diagram will help you get familiar with the layout of the board and get started in immersing yourself into your own passion projects.

The Raspberry Pi is a series of credit card sized single-board computers developed in the United Kingdom by the Raspberry Pi Foundation to promote the teaching of basic computer science in schools and developing countries.

Raspberry Pi 2 includes a quad-core Cortex-A7 CPU running at $900 \mathrm{MHz}$ and 1 GB RAM. It is described as 4-6 times more powerful than its predecessor. The GPU is identical to the original. The Raspberry Pi does not have a built-in real time clock, and does not "know" the time of day. It have many models All models feature a Broadcom system on a chip (SoC), which includes an ARM compatible central processing unit (CPU) and an on chip graphics processing unit (GPU, a Video Core IV).

CPU speed ranges from $700 \mathrm{MHz}$ to $1.2 \mathrm{GHz}$ for the Pi 3 and on board memory range from 256 MB to 1 GB RAM.

Secure Digital (SD) cards are used to store the operating system and program memory in either the SDHC or Micro SDHC sizes. Most boards have between one and four USB slots, HDMI and composite video output, and a $3.5 \mathrm{~mm}$ phone jack for audio.

\section{Models of Raspberry Pi}

Five models:

Raspberry Pi 1 Model B

Raspberry Pi 1 Model B+. 
Raspberry Pi Zero

RaspberryPi2

RaspberryPi3ModelB

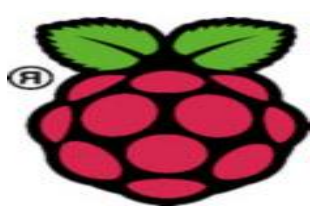

\section{Raspberry Pi 3 Model B}

The Raspberry Pi 3 is the third generation Raspberry Pi. It replaced the Raspberry Pi 2 Model B in February 2016.

We recommend the Raspberry Pi 3 Model B for use in schools, or for any general use. Those wishing to embed their $\mathrm{Pi}$ in a project may prefer the Pi zero or model A+, which are more useful for embedded projects, and projects which require very low power.

The Raspberry Pi 2 and the Raspberry Pi 3 have 1 GB of RAM

The Raspberry Pi 3 is equipped with $2.4 \mathrm{GHz}$ WI-Fi 802.11n (150 Mbit/s) and Bluetooth 4.1 (24 Mbit/s) in addition to the $10 / 100$ Ethernet port.

Although the Raspberry Pi 3 does not have H.265 decoding hardware, the CPU, more powerful than its predecessors, is potentially able to decode H.265-encoded videos in software. The Open Source Media Center (OSMC) project said in February 2016.

Raspberry Pi image for Raspberry Pi 2 and the new Raspberry Pi 3. Only when 64-bit support is ready, and beneficial to OSMC users, will we offer a separate image.

\section{Features of Raspberry Pi Model 3 B}

A 1.2GHz 64-bit quad-core ARMv8 CPU

802.11n Wireless LAN

Bluetooth 4.1

Bluetooth Low Energy (BLE) 1GB RAM

4 USB ports

40 GPIO pins

Full HDMI port

Ethernet port

Combined $3.5 \mathrm{~mm}$ audio jack and composite video

Camera interface (CSI)

Display interface (DSI)

Micro SD card slot (now push-pull rather than push-push)

Video Core IV 3D graphics core 


\section{Results and Discussions}

Face of the student is recognized and after that the online page open. The online webpage will be counting the question.

\section{OpenCV}

OpenCV (Open Source Computer Vision Library) is an open source computer vision and machine learning software library. OpenCV was built to provide a common infrastructure for computer vision applications and to accelerate the use of machine perception in the commercial products. Being a BSD-licensed product, OpenCV makes it easy for businesses to utilize and modify the code.

The library has more than 2500 optimized algorithms, which includes a comprehensive set of both classic and state-of-the-art computer vision and machine learning algorithms. These algorithms can be used to detect and recognize faces, identify objects, classify human actions in videos, track camera movements, track moving objects, extract 3D models of objects, produce 3D point clouds from stereo cameras, stitch images together to produce a high resolution image of an entire scene, find similar images from an image database, remove red eyes from images taken using flash, follow eye movements, recognize scenery and establish markers to overlay it with augmented reality, etc. OpenCV has more than 47 thousand people of user community and estimated number of downloads exceeding.

\section{Python}

The Python programming language actually started as a scripting language for Linux. Python programs are similar to shell scripts in that the files contain a series of commands that the computer executes from top to bottom. Python is a very useful and versatile high level programming language, with easy to read syntax that allows programmers to use fewer lines of code than would be possible in languages such as assembly, C, or Java.

Python programs don't need to be compiled before running them, as you do with C programs. However, you will need to install the Python interpreter on your computer to run them. The interpreter is the program that reads the Python file and executes the code. There are programs like or $r$ that can package Python code into stand-alone executable programs so you can run Python programs on computers without the Python interpreter installed.

Like shell scripts, Python can automate tasks like batch renaming and moving large amounts of files. Using IDLE, Python's REPL (read, eval, print, loop) function can be used just like a command line. However, there are more useful things you can create with Python. Programmers use Python to create things like:

Web applications

Desktop applications and utilities

Special GUIs

Small databases

2D games 


\section{Conclusions and Recommendations}

Here, we written the question paper in PHP format. The PHP page is in the web server. Whenever the face of student is matching with the database then only the page will be opened. Otherwise, if the person detected is unauthorized person then output screen will show it as not recognised. Thus, only the student can appear the exam and the malpractices can be avoided.

\section{Acknowledgements}

People who contributed to the work but do not fit criteria for authorship are our all teachers as well as parents.

\section{References}

[1] S. Kafkas, and Z. Bayram, "Distance education using XML technology", Information Technology Based Higher Education and Training, IEEE Proceedings of the Fifth International Conference, 2004.

[2] L. Yu-Chun, Y. Yu-Sheng, C. Ping-Chun, and Y. Chu-Sing, "The design and implementation of intelligent assessment management system", Global Engineering Education Conference, IEEE, 2013.

[3] T. Prasad, and A. Acharya, "An Architecture of Cloud Computing Based Online Examination System", IJCST, vol. 3, pp. 524-526, 2012.

[4] G. Ganesh Sriram, B.VijayaAditya, M.Gupta, R.Santosh Kumar, "Application Study on Cloud Computing Based Virtual Campus", IJIET, vol. 2, pp. 357-361, 2013.

[5] A. Crasto1, S. K. Sinha, S. Raut, and I. Mirza, "Ubiquitous Computing in a Lab Environment", IEEE International Conference, 2013.

[6] Z. A. Hua, "Study of Ubiquitous Learning Environment Based on Ubiquitous Computing", IEEE International Conference, 2010.

[7] S. G. Deshpande, and J. N. Hwang, "A Real Time Interactive Virtual Classroom Multimedia Distance Learning System", IEEE International Conference, 2001.

[8] C. A. Romney, "Tablet PC Use in Freshman Mathematics Classes Promotes STEM Retention", IEEE International Conference, 2011.

*Corresponding author.

E-mail address: Kunalpawar309@ gmail.com 